\title{
Stackelberg Game Modeling of Pricing for Mobile Virtual Network Operators
}

\author{
Yali Wang ${ }^{\dagger}$ \\ Institute of Intelligent Grid \\ Technology \\ School of Electrical and \\ Electronic Engineering, North \\ China Electric Power \\ University \\ 102206, Beijing, China \\ wangyali@ncepu.edu.cn
}

\author{
Bo $\mathrm{Gu}$ \\ Institute of Intelligent Grid \\ Technology \\ School of Electrical and \\ Electronic Engineering, North \\ China Electric Power \\ University \\ 102206, Beijing, China \\ coopertwo@gmail.com
}

\author{
Song Liu \\ Institute of Intelligent Grid \\ Technology \\ School of Electrical and \\ Electronic Engineering, North \\ China Electric Power \\ University \\ 102206, Beijing, China \\ liusong@ncepu.edu.cn
}

\author{
Peng Liu \\ Institute of Intelligent Grid \\ Technology \\ School of Electrical and \\ Electronic Engineering, North \\ China Electric Power \\ University \\ 102206, Beijing, China \\ liupengwhite@gamil.com
}

\author{
Xiao Zhong \\ Institute of Intelligent Grid \\ Technology \\ School of Electrical and \\ Electronic Engineering, North \\ China Electric Power \\ University \\ 102206, Beijing, China \\ zhongxiao@ncepu.edu.cn
}

\begin{abstract}
With rapid development of new network technologies, demands of network users grow dramatically. Primary mobile network operators (POs) cannot meet the demands of users. Therefore, secondary mobile virtual network operators (SOs) emerge to alleviate pressure on the network market. Obviously, the competition becomes more serious, for users have more choices for operators based on their requirements. Because all operators want to get their maximum profits, the main research of this paper is to obtain the maximum profits for one PO and one SO through pricing which uses Stackelberg leader-follower game. We have considered the changes of users' demands, got the real-time equilibrium pricing point, and finally proved the correctness of our method.
\end{abstract}

\section{Keywords}

\footnotetext{
* This study was supported by State Key Laboratory of Alternate Electrical Power System with Renewable Energy Sources (Grant No. LAPS14015) and "the Fundamental Research Funds for the Central Universities".

${ }^{\dagger}$ Miss. Wang insisted her name be first and did the really hard work.
}

PO, SO, Pricing, Network users, Stackelberg game, Realtime equilibrium pricing point

\section{INTRODUCTION}

Wireless spectrum can be obtained in almost all countries all over the world, the utilization of wireless spectrum is indispensable for all businesses of wireless communications. The wireless spectrum refers to the continuous frequencies ranging from $3 \mathrm{kHz}$ to $300 \mathrm{GHz}$ typically. Concerning the management of wireless spectrum [1,2], spectrum management organizations for all countries have common characteristics. They divide wireless spectrum into continuous frequency bands. Each frequency band will be allocated to a particular primary mobile network operator (PO), and these operators will be granted absolute ownership for the given frequency bands [3]. Other operators who don't get the license rights can't directly employ the given frequency bands. However, once an operator gets the spectrum license right, it possesses the right to lease portion of its spectrum to other operators, who also wish to entering into network market. This phenomenon can improve the social benefit [4].

Many articles have investigated the pricing competition in wireless network and game theory $[5,6,7,8]$. Luis Grijarro establishes a model about the competition between one PO and one SO [9]. He adopts a method of backward induction to analysis the interaction between $\mathrm{PO}$ and SO. Our paper is based on his model. We use a Stackelberg leader-follower game to resolve the competition game, and give the specific calculations for readers.

In this paper, the problem of how to maximize the profits of one primary mobile network operator (PO) and one secondary mobile virtual network operator (SO) through pric- 
ing is analyzed. We establish a model about the relationship of $\mathrm{PO}, \mathrm{SO}$ and users, and use Stackelberg game to resolve the problem above [10]. In order to develop technology innovation, $\mathrm{PO}$ leases portion of its spectrum license rights to SO $[11,12]$. Both PO and SO provide service for users. Users choose PO or SO based on price, QoS (quality of service) and their basic willingness to pay [13]. In this paper, It is assumed that there are only two operators in the network market, $\mathrm{PO}$ and $\mathrm{SO}$ respectively, and users have the right to choose one operator or neither.

The main contributions of this paper are as follows:

(1) Analyze the change of numbers which users choose PO and SO through considering the distribution of users' basic willingness to pay for each bandwidth.

(2) Maximize the profits of PO and SO through pricing using Stackelberg game.

(3) Obtain the relationship between the equilibrium price point and users' demands and network supplies.

The remainder of this paper is organized as follows. Service model, users' utilities and definition of operators' profits are described in Section 2. Specific calculations process of game is derived in Section 3. Results of Stackelberg game are analyzed in Section 4. Section 5 concludes this paper and introduces the future work. The final part is the references.

\section{SERVICE MODEL}

The service model is depicted in Fig. 1. It is composed of $\mathrm{PO}, \mathrm{SO}$ and users. We assume that there are only two network operators in the network market. PO possesses the spectrum license rights of $W \mathrm{MHz}$ frequency bandwidth in total, and leases $b \mathrm{MHz}$ to $\mathrm{SO}$, which means that it leaves itself $W-b \mathrm{MHz}$. SO pays for spectrum licenses at a price of $p$ per Hz. Both $\mathrm{PO}$ and SO provide service to users. Each user should pay $p_{\mathrm{p}}$ m.u. if he chooses PO, or pay $p_{\mathrm{s}}$ m.u. if he chooses SO. Here, m.u. is monetary unit. These three prices $p, p_{\mathrm{p}}$ and $p_{\mathrm{s}}$ are referred at the same time period. We assume that there are $n$ users in total, and the quantity of users is enough for competition. There are $n_{\mathrm{p}}$ users who choose PO and $n_{\mathrm{s}}$ users who choose $\mathrm{SO}$ at state which users do not change their demands. It is assumed that the number of users who choose PO at next state which users' demands changed is $n * \Omega_{\mathrm{p}}$, and for $\mathrm{SO}$ is $n * \Omega_{\mathrm{s}} . \Omega_{\mathrm{p}}$ is the percent number of users who choose PO, so as $\Omega_{\mathrm{s}}$. We study the moment which users' demands change, so the unit of time is $1 \mathrm{~s}$.

Users' utilities and definition of operators' profits will be represented in 2.1 and 2.2 .

\subsection{Users' Utilities}

There are three factors which may influence one user's choice: user's basic willingness to pay for each bandwidth, QoS, and price respectively.

Firstly, this paper defines $\tau_{i}$ as user's basic willingness to pay for each bandwidth. $i=[1,2, \ldots, \mathrm{n}] . \tau_{i}$ is distributed uniformly. It's probability density function $f(x)$ is positive and continuous on $[0, \phi]$ and $\phi>0$. It's cumulative density function is defined as $F(a)=\int_{-\infty}^{a} f(x) d x$ for all $a \in R$. The unit of $\tau_{i}$ is m.u./bit. We assume that users' demands change at the first second on the next state.

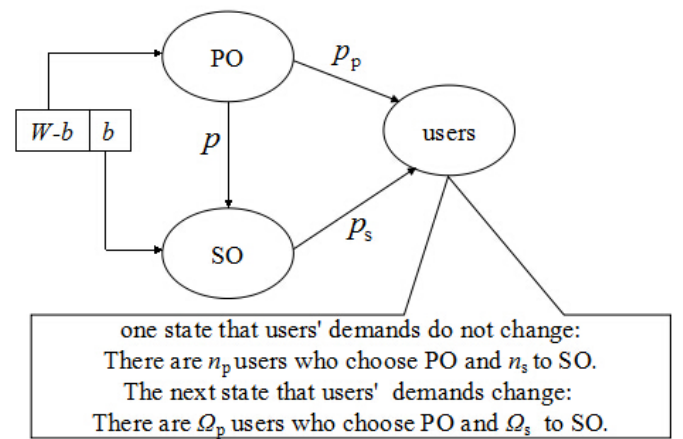

Figure 1: Service model.

Secondly, this paper defines $Q_{\mathrm{p}}$ and $Q_{\mathrm{s}}$ as the representative of QoS which is offered by $\mathrm{PO}$ and $\mathrm{SO}$, respectively. $Q_{\mathrm{p}}$ and $Q_{\mathrm{s}}$ can be described by spectral efficiencies $k^{(\mathrm{p})}$ and $k^{(\mathrm{s})}$ [14]. $k^{(\mathrm{p})}$ and $k^{(\mathrm{s})}$ are assumed fixed and $k^{(\mathrm{p})}<k^{(\mathrm{s})}$. SO has a more efficient operation than PO, thus it can compete with PO. The spectrum supplied by each operator is evenly divided by users, so QoS can be influenced by $n_{\mathrm{p}}$ and $n_{\mathrm{s}}$. The more users choose PO, the worse QoS becomes. While QoS becomes worse, less users will choose PO. The functions of $Q_{\mathrm{p}}$ and $Q_{\mathrm{s}}$ are described as $Q_{\mathrm{p}}=\log \left(\frac{k^{(\mathrm{p})}(W-b)}{n_{\mathrm{p}}}\right), Q_{\mathrm{s}}=$ $\log \left(\frac{k^{(\mathrm{s})} b}{n_{\mathrm{s}}}\right)$. This indicates that the QoS is affected by supplies and demands. We define that $Q_{\mathrm{p}}>Q_{\mathrm{s}}$ for PO has more resources than $\mathrm{SO}$.

The third factor is price. The higher the price is, the lower the utility is. Users intend to get high quality of service with low price.

Considering the discussions above, user's utility functions are obtained. We use $u_{i, \mathrm{p}}$ and $u_{i, \mathrm{~s}}$ to represent the utility functions for PO and SO individually.

$$
\begin{aligned}
& u_{i, \mathrm{p}}=\tau_{i} * Q_{\mathrm{p}}-p_{\mathrm{p}} \\
& u_{i, \mathrm{~s}}=\tau_{i} * Q_{\mathrm{s}}-p_{\mathrm{s}}
\end{aligned}
$$

The unit of $Q_{\mathrm{p}}$ is bit, so the unit of $u_{i, \mathrm{p}}$ is m.u.. Users expect to obtain corresponding or more services for their net charge. Therefore, user $i$ chooses PO when $u_{i, \mathrm{p}}>u_{i, \mathrm{~s}}$ and $u_{i, \mathrm{p}}>0$, chooses SO when $u_{i, \mathrm{p}}<u_{i, \mathrm{~s}}$ and $u_{i, \mathrm{~s}}>0$, and chooses neither when $u_{i, \mathrm{p}}<0$ and $u_{i, \mathrm{~s}}<0$.

\subsection{Definition of Operators' Profits}

In this paper, the expressions of the profits of $\mathrm{PO}$ and $\mathrm{SO}$ are described as

$$
\begin{gathered}
\Pi_{\mathrm{p}}=n * \Omega_{\mathrm{p}} * p_{\mathrm{p}}+p * b-C_{\mathrm{p}} \\
\Pi_{\mathrm{s}}=n * \Omega_{\mathrm{s}} * p_{\mathrm{s}}-p * b-C_{\mathrm{s}}
\end{gathered}
$$

$\mathrm{PO}$ can get profits from users and SO. $\Omega_{\mathrm{p}}$ is the percent number of users who choose PO after our game, $p_{\mathrm{p}}$ is the net charge that each user should pay. SO pays the rent to PO. SO buys $b \mathrm{MHz}$ spectrum license rights from PO. $p$ is the unit price. $C_{\mathrm{p}}$ is the basic cost of PO. The composition of the profit of $\mathrm{SO}$ is similar. 
Table 1: Basic notations used in our game model

\begin{tabular}{|c|c|c|}
\hline notation & unit & The meaning of each notation \\
\hline$W$ & $\mathrm{MHz}$ & $\begin{array}{l}\text { Total amount of the spectrum } \\
\text { of PO }\end{array}$ \\
\hline$b$ & $\mathrm{MHz}$ & $\begin{array}{l}\text { Amount of the spectrum that } \\
\text { PO lease to SO }\end{array}$ \\
\hline$p$ & m.u./Hz & $\begin{array}{l}\text { Price that PO leases to } \mathrm{SO} \\
\text { for per } \mathrm{Hz}\end{array}$ \\
\hline$p_{\mathrm{p}}$ & m.u. & Money that users should pay to PO \\
\hline$p_{\mathrm{s}}$ & m.u. & Money that users should pay to SO \\
\hline$n$ & 1 & Total amount of users in this paper \\
\hline$n_{\mathrm{p}}$ & 1 & $\begin{array}{l}\text { Amount of users who choose } \\
\text { PO before our game }\end{array}$ \\
\hline$n_{\mathrm{s}}$ & 1 & $\begin{array}{l}\text { Amount of users who choose } \\
\text { SO before our game }\end{array}$ \\
\hline$\tau_{i}$ & m.u./bit & $\begin{array}{l}\text { Basic willingness that users } \\
\text { want to pay for each bit }\end{array}$ \\
\hline$\phi$ & m.u./bit & $\begin{array}{l}\text { Upper limit of users' basic } \\
\text { willingness to pay }\end{array}$ \\
\hline$Q_{\mathrm{p}}$ & bit & $\begin{array}{l}\text { Quality factor which describes } \\
\text { the quality of the service of } \mathrm{PO}\end{array}$ \\
\hline$Q_{\mathrm{s}}$ & bit & $\begin{array}{l}\text { Quality factor which describes } \\
\text { the quality of the service of SO }\end{array}$ \\
\hline$k^{(\mathrm{p})}$ & $\mathrm{bit} / \mathrm{s} / \mathrm{Hz}$ & Spectral efficiency for PO \\
\hline$k^{(\mathrm{s})}$ & $\mathrm{bit} / \mathrm{s} / \mathrm{Hz}$ & Spectral efficiency for SO \\
\hline$u_{i, \mathrm{p}}$ & m.u. & Utility of each user i to $\mathrm{PO}$ \\
\hline$u_{i, \mathrm{~s}}$ & m.u. & Utility of each user i to SO \\
\hline$\tau_{i, \mathrm{p}}$ & m.u./bit & Critical point of $\tau_{i}$ when $u_{i, \mathrm{p}}=0$ \\
\hline$\tau_{i, \mathrm{~s}}$ & m.u./bit & Critical point of $\tau_{i}$ when $u_{i, \mathrm{~s}}=0$ \\
\hline$\tau_{i}^{0}$ & m.u./bit & Critical point of $\tau_{i}$ when $u_{i, \mathrm{p}}=u_{i, \mathrm{~s}}$ \\
\hline$\Pi_{\mathrm{p}}$ & m.u. & Profit of PO \\
\hline$\Pi_{\mathrm{s}}$ & m.u. & Profit of SO \\
\hline$C_{\mathrm{p}}$ & m.u. & Basic cost of $\mathrm{PO}$ \\
\hline$C_{\mathrm{s}}$ & m.u. & Basic cost of SO \\
\hline$\Omega_{\mathrm{p}}$ & 1 & $\begin{array}{l}\text { Percent of users who choose PO } \\
\text { after considering users' basic } \\
\text { willingness to pay for each bit } \\
\text { Percent of users who choose SO }\end{array}$ \\
\hline$\Omega_{\mathrm{s}}$ & 1 & $\begin{array}{l}\text { after considering users' basic } \\
\text { willingness to pay for each bit }\end{array}$ \\
\hline$p_{\mathrm{p}}^{*}$ & m.u. & Equilibrium price of $\mathrm{PO}$ after game \\
\hline$p_{\mathrm{s}}^{*}$ & m.u. & Equilibrium price of SO after game \\
\hline
\end{tabular}

We assume that $p, b, C_{\mathrm{p}}$ and $C_{\mathrm{s}}$ are fixed in this paper. But at the future work, $p$ and $b$ will be considered to affect the profit of operators.

The notions used throughout this paper are summarized as shown in Table 1.

\section{SPECIFIC PROCESS OF GAME}

The general process of our game can be summarized in two steps. First, we should calculate the number of users who choose two operators through considering the distribution of their basic willingness to pay for each bandwidth. The second step is to maximize the profits of two operators through Stackelberg game.

\subsection{Users' Choices}
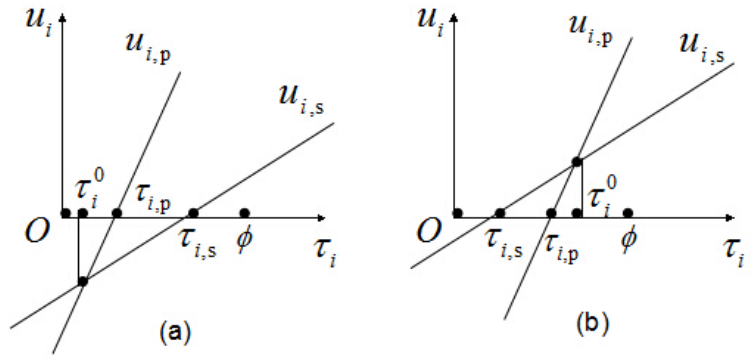

(b)

Figure 2: The utility function for $\tau_{i}$.

There are three important points for the utility. Considering $u_{i, \mathrm{p}}$ and $u_{i, \mathrm{~s}}$ as the functions of $\tau_{i} . Q_{\mathrm{p}}$ is known as $W, b$ and $n_{\mathrm{p}}$ are known information. $n_{\mathrm{p}}$ reflects the demands of users and it is known information in our game. When $u_{i, \mathrm{p}}=0$, we can get that $\tau_{i, \mathrm{p}}=\frac{p_{\mathrm{p}}}{Q_{\mathrm{p}}}$. When $u_{i, \mathrm{~s}}=0, \tau_{i, \mathrm{~s}}=\frac{p_{\mathrm{s}}}{Q_{\mathrm{s}}}$. And when $u_{i, \mathrm{p}}=u_{i, \mathrm{~s}}, \tau_{i}^{0}=\frac{p_{\mathrm{p}}-p_{\mathrm{s}}}{Q_{\mathrm{p}}-Q_{\mathrm{s}}}$. For $Q_{\mathrm{p}}>Q_{\mathrm{s}}$, the slope of users' utilities who choose PO and $\mathrm{SO}$ can be got. If $\tau_{i, \mathrm{p}}<\tau_{i, \mathrm{~s}}$, we can get that $\tau_{i}^{0}<\tau_{i, \mathrm{p}}<\tau_{i, \mathrm{~s}}$ for $\frac{Q_{\mathrm{s}}}{Q_{\mathrm{p}}-Q_{\mathrm{s}}}>0$. If $\tau_{i, \mathrm{~s}}<\tau_{i, \mathrm{p}}$, $\tau_{i, \mathrm{~s}}<\tau_{i, \mathrm{p}}<\tau_{i}^{0}$ can be got. These can be proved furthermore by Fig. 2 . When $u_{i, \mathrm{p}}=u_{i, \mathrm{~s}}>0$, we can get the game equilibrium. So the condition that $\tau_{i, \mathrm{~s}}<\tau_{i, \mathrm{p}}<\tau_{i}^{0}$ is reasonable. We have known that $\tau_{i} \in[0, \phi]$, so $0<\tau_{i, \mathrm{~s}}<\tau_{i, \mathrm{p}}<\tau_{i}^{0}<\phi$. It can be obtained the distribution of users through analyzing Fig. 2(b).

$$
\begin{gathered}
\Omega_{\mathrm{p}}=P\left(\tau_{i}^{0}<\tau_{i}<\phi\right)=1-\frac{\tau_{i}^{0}}{\phi}=1-\frac{p_{\mathrm{p}}-p_{\mathrm{s}}}{\phi\left(Q_{\mathrm{p}}-Q_{\mathrm{s}}\right)} \\
\Omega_{\mathrm{s}}=P\left(\tau_{i, \mathrm{~s}}<\tau_{i}<\tau_{i}^{0}\right)=\frac{\tau_{i}^{0}}{\phi}-\frac{\tau_{i, \mathrm{~s}}}{\phi}=\frac{p_{\mathrm{p}}-p_{\mathrm{s}}}{\phi\left(Q_{\mathrm{p}}-Q_{\mathrm{s}}\right)}-\frac{p_{\mathrm{s}}}{\phi Q_{\mathrm{s}}}
\end{gathered}
$$

When $\tau_{i}^{0}<\tau_{i}<\phi$, it can be got that $u_{i, \mathrm{p}}>u_{i, \mathrm{~s}}$. When $\tau_{i, \mathrm{~s}}<\tau_{i}<\tau_{i}^{0}, u_{i, \mathrm{p}}<u_{i, \mathrm{~s}} . \Omega_{\mathrm{p}}$ and $\Omega_{\mathrm{s}}$ are the percent number which users choose $\mathrm{PO}$ or $\mathrm{SO}$ and they are the functions of $p_{\mathrm{p}}$ and $p_{\mathrm{s}}$. Therefore, the profit of each operator is the function of $p_{\mathrm{p}}$ and $p_{\mathrm{s}}$. If we adopt the fixed price, the number of users who choose PO is $n^{*} \Omega_{\mathrm{p}}$, so as SO which is $n^{*} \Omega_{\mathrm{s}}$, and they are also fixed. Because we want to obtain the maximum profits of two operators, we use game theory to change the price to get an equilibrium point. That is the reason why we consider $\Omega_{\mathrm{p}}, \Omega_{\mathrm{s}}, \Pi_{\mathrm{p}}$ and $\Pi_{\mathrm{s}}$ as the functions of $p_{\mathrm{p}}$ and $p_{\mathrm{s}}$.

\subsection{Pricing Game}

In this paper, Stackelberg leader-follower game is used to obtain the maximum profits of $\mathrm{PO}$ and $\mathrm{SO}$. PO is the leader, and $\mathrm{SO}$ is the follower. PO makes its strategy first, and then SO makes the corresponding strategy to maximize its own profit. PO knows what strategies SO would make. The specific analysis using mathematics is as follow:

Firstly, assuming that $p_{\mathrm{p}}$ is fixed, so that $\Pi_{\mathrm{s}}$ is only the function of $p_{\mathrm{s}}$. Then we calculate the partial deriving for $\Pi_{\mathrm{s}}$ to $p_{\mathrm{s}}$. Let the partial derivative function equal to zero, then the best response of $\mathrm{SO}$ is got. $p_{\mathrm{p}}$ is expressed by $p_{\mathrm{s}}$. 
Next, we substitute $p_{\mathrm{s}}$ into the function of $\Pi_{\mathrm{p}} . \Pi_{\mathrm{p}}$ will be the function of $p_{\mathrm{p}}$ only. So the maximum profit of $\Pi_{\mathrm{p}}$ can be got through deriving $\Pi_{\mathrm{p}}$ to $p_{\mathrm{p}}$ and let this equal to zero. The equilibrium pricing point is obtained. Finally, the maximum values of both $\Pi_{\mathrm{p}}$ and $\Pi_{\mathrm{s}}$ are also got. The specific process of the calculations are as follows:

Calculating partial deriving for $\Pi_{\mathrm{s}}$ to $p_{\mathrm{s}}$ and let it equal to zero. They can be shown as Eq. (7) and Eq. (8):

$$
\begin{gathered}
\Pi_{\mathrm{s}}=n *\left(\frac{p_{\mathrm{p}}-p_{\mathrm{s}}}{\phi\left(Q_{\mathrm{p}}-Q_{\mathrm{s}}\right)}-\frac{p_{\mathrm{s}}}{\phi Q_{\mathrm{s}}}\right) * p_{\mathrm{s}}-p * b-C_{\mathrm{s}} \\
\frac{\partial \Pi_{\mathrm{s}}}{\partial p_{\mathrm{s}}}=0
\end{gathered}
$$

$p_{\mathrm{s}}$ is expressed as the function of $p_{\mathrm{p}}$ base on our mathematical calculation.

$$
p_{\mathrm{s}}=\frac{Q_{\mathrm{s}}}{2 Q_{\mathrm{p}}} * p_{\mathrm{p}}
$$

Next, by substituting $p_{\mathrm{s}}$ into the function of $\Pi_{\mathrm{p}}$, we can express $\Pi_{\mathrm{p}}$ as the function of $p_{\mathrm{p}}$ only. Furthermore, we make the derivation for $\Pi_{\mathrm{p}}$ to $p_{\mathrm{p}}$, and let the derived function equal to zero. They can be expressed as Eq. (10) and Eq. (11).

$$
\begin{gathered}
\Pi_{\mathrm{p}}=n *\left(1-\frac{p_{\mathrm{p}}-\frac{Q_{\mathrm{s}}}{2 Q_{\mathrm{p}}} * p_{\mathrm{p}}}{\phi\left(Q_{\mathrm{p}}-Q_{\mathrm{s}}\right)}\right) * p_{\mathrm{p}}+p * b-C_{\mathrm{p}} \\
\frac{d \Pi_{\mathrm{p}}}{d p_{\mathrm{p}}}=0
\end{gathered}
$$

$p_{\mathrm{p}}$ can be got as Eq. (12)

$$
p_{\mathrm{p}}^{*}=\frac{\phi\left(Q_{\mathrm{p}}-Q_{\mathrm{s}}\right)}{2-\frac{Q_{\mathrm{s}}}{Q_{\mathrm{p}}}}
$$

Furthermore, $p_{\mathrm{s}}$ can be got as Eq. (13)

$$
p_{\mathrm{s}}^{*}=\frac{\phi\left(Q_{\mathrm{p}}-Q_{\mathrm{s}}\right) Q_{\mathrm{s}}}{2\left(2-\frac{Q_{\mathrm{s}}}{Q_{\mathrm{p}}}\right) Q_{\mathrm{p}}}
$$

According to Eq. (12) and Eq. (13), the value of $p_{\mathrm{p}}$ and $p_{\mathrm{s}}$ can be got. Therefore the max values of $\Pi_{\mathrm{p}}$ and $\Pi_{\mathrm{s}}$ are obtained from Eq. (3) and Eq. (4) under the situation that variables $p, b, C_{\mathrm{p}}$ and $C_{\mathrm{s}}$ are known. Section 4 will analyzes our results.

\section{ANALYSIS RESULTS}

In this paper, we analyze the results from two aspects: 1) The relationship between the equilibrium price point and users' demands and network supplies. 2) Demonstration of the correctness of our method.

\subsection{Analysis of Equilibrium Point}

It can be proved that the equilibrium price $p_{\mathrm{p}}^{*}$ and $p_{\mathrm{s}}^{*}$ are changed with users' demands and network supplies. $p_{\mathrm{p}}^{*}$ and $p_{\mathrm{s}}^{*}$ relate to $Q_{\mathrm{p}}$ and $Q_{\mathrm{s}}$ from Eq. (12) and Eq. (13). But $Q_{\mathrm{p}}$ and $Q_{\mathrm{s}}$ are the functions of $W, b, n_{\mathrm{p}}$ and $n_{\mathrm{s}}$ which are the specific reflections of supplies and demands.

$p_{\mathrm{p}}^{*}$ and $p_{\mathrm{s}}^{*}$ are the linear functions for $\phi$, and they are strictly monotone increasing with $\phi$. That is, the higher users' maximum willingness to pay for each bandwidth, the higher the price. It can be proved through $\frac{Q_{\mathrm{p}}-Q_{\mathrm{s}}}{2-\frac{Q_{\mathrm{s}}}{Q_{\mathrm{p}}}}>0$ as $Q_{\mathrm{p}}>Q_{\mathrm{s}}$.

As Eq. (12) shows, the higher the $Q_{\mathrm{p}}$, the higher the $p_{\mathrm{p}}^{*}$. Considering that $p_{\mathrm{p}}^{*}$ as the function of $Q_{\mathrm{p}}$, we can validate our conclusion as Eq. (14)

$$
\frac{d p_{\mathrm{p}}^{*}}{d Q_{\mathrm{p}}}=\frac{\phi\left(1+\left(1-\frac{Q_{\mathrm{s}}}{Q_{\mathrm{p}}}\right)^{2}\right)}{\left(2-\frac{Q_{\mathrm{s}}}{Q_{\mathrm{p}}}\right)^{2}}>0
$$

In the same way, $p_{\mathrm{p}}^{*}$ decreases with the increase of $Q_{\mathrm{s}}$ :

$$
\frac{d p_{\mathrm{p}}^{*}}{d Q_{\mathrm{s}}}=\frac{-\phi}{\left(2-\frac{Q_{\mathrm{s}}}{Q_{\mathrm{p}}}\right)^{2}}<0
$$

Considering $p_{\mathrm{s}}^{*}$ as the function of $Q_{\mathrm{p}}$ :

$$
\frac{d p_{\mathrm{s}}^{*}}{d Q_{\mathrm{p}}}=\frac{\phi Q_{\mathrm{s}}^{2}}{2\left(2-\frac{Q_{\mathrm{s}}}{Q_{\mathrm{p}}}\right)^{2} Q_{\mathrm{p}}^{2}}>0
$$

Finally considering $p_{\mathrm{s}}^{*}$ as the function of $Q_{\mathrm{s}}$ :

$$
\frac{d p_{\mathrm{s}}^{*}}{d Q_{\mathrm{s}}}=\frac{\phi\left((2+\sqrt{2}) Q_{\mathrm{p}}-Q_{\mathrm{s}}\right)}{2\left(2-\frac{Q_{\mathrm{s}}}{Q_{\mathrm{p}}}\right)^{2} Q_{\mathrm{p}}^{2}} *\left((2-\sqrt{2}) Q_{\mathrm{p}}-Q_{\mathrm{s}}\right)
$$

We have known that $Q_{\mathrm{p}}>Q_{\mathrm{s}}$, so that $\frac{\phi\left((2+\sqrt{2}) Q_{\mathrm{p}}-Q_{\mathrm{s}}\right)}{2\left(2-\frac{Q_{\mathrm{s}}}{Q_{\mathrm{p}}}\right)^{2} Q_{\mathrm{p}}^{2}}>0$. There is a point that $Q_{\mathrm{s}}=(2-\sqrt{2}) Q_{\mathrm{p}}$ when $\frac{d p_{\mathrm{s}}^{*}}{d Q_{\mathrm{s}}}=0 . p_{\mathrm{s}}^{*}$ increases when $0<Q_{\mathrm{s}}<(2-\sqrt{2}) Q_{\mathrm{p}}$, but decreases when $(2-\sqrt{2}) Q_{\mathrm{p}}<Q_{\mathrm{s}}<Q_{\mathrm{p}}$ as $Q_{\mathrm{s}}$ increases.

Eq. (17) reflects that the price should be decreased when the supply is overmuch. Those above analyze the relationship between the equilibrium price and users' demands and network supplies. It shows that our equilibrium pricing point is real-time with the supplies and demands. The correctness of our method will be proved next.

\subsection{Maximum Profit of Operators}

In this paper, both $\mathrm{PO}$ and $\mathrm{SO}$ get their maximum profits at the Stackelberg equilibrium point. $\Pi_{\mathrm{s}}\left(p_{\mathrm{p}}^{*}, p_{\mathrm{s}}^{*}\right)>\Pi_{\mathrm{s}}\left(p_{\mathrm{p}}^{*}, p_{\mathrm{s}}\right)$, $\Pi_{\mathrm{p}}\left(p_{\mathrm{p}}^{*}, p_{\mathrm{s}}^{*}\right)>\Pi_{\mathrm{p}}\left(p_{\mathrm{p}}, \frac{Q_{\mathrm{s}}}{2 Q_{\mathrm{p}}} p_{\mathrm{p}}\right)$, The specific expressions of $\Pi_{\mathrm{s}}\left(p_{\mathrm{p}}^{*}, p_{\mathrm{s}}\right)$ and $\Pi_{\mathrm{p}}\left(p_{\mathrm{p}}, \frac{Q_{\mathrm{s}}}{2 Q_{\mathrm{p}}} p_{\mathrm{p}}\right)$ are shown as Eq. (18) and Eq. (19). The equations can be imaged as Fig. 3 and Fig. 4.

$$
\Pi_{\mathrm{s}}\left(p_{\mathrm{p}}^{*}, p_{\mathrm{s}}\right)=n *\left(\frac{p_{\mathrm{p}}^{*}-p_{\mathrm{s}}}{\phi\left(Q_{\mathrm{p}}-Q_{\mathrm{s}}\right)}-\frac{p_{\mathrm{s}}}{\phi Q_{\mathrm{s}}}\right) * p_{\mathrm{s}}-p * b-C_{\mathrm{s}}
$$

$\Pi_{\mathrm{p}}\left(p_{\mathrm{p}}, \frac{Q_{\mathrm{s}}}{2 Q_{\mathrm{p}}} * p_{\mathrm{p}}\right)=n *\left(1-\frac{p_{\mathrm{p}}-\frac{Q_{\mathrm{s}}}{2 Q_{\mathrm{p}}} p_{\mathrm{p}}}{\phi\left(Q_{\mathrm{p}}-Q_{\mathrm{s}}\right)}\right) * p_{\mathrm{p}}+p * b-C_{\mathrm{p}}$

Eq. (18), Eq. (19), Fig. (3) and Fig. (4) prove the correctness of our method that using Stackelberg game can maximize operators' profits. According to Eq. (18), we can realize that $\mathrm{SO}$ gets its best reaction with PO's price to acquire its maximum profit. We can also realize that $\mathrm{PO}$ can obtain its best strategy from Eq. (19) as PO has known SO's response. If SO does't take its best response as Eq. (9), it can't get the maximum profit. In the same way, if SO takes the best strategy while PO has't chosen the price $p_{\mathrm{p}}^{*}$, PO also can't obtain the maximum profit. 


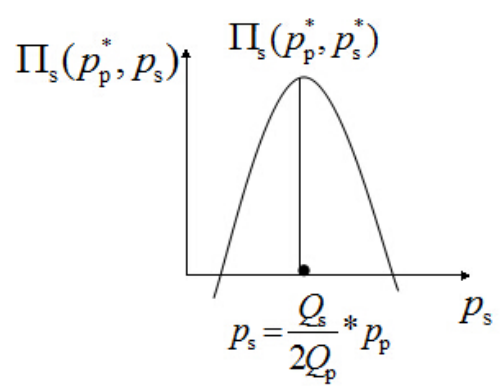

Figure 3: The expression of $\Pi_{\mathbf{s}}\left(p_{\mathbf{p}}^{*}, p_{\mathbf{s}}\right)$.

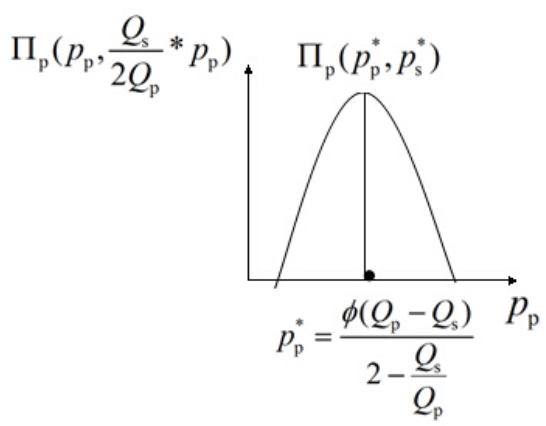

Figure 4: The expression of $\Pi_{\mathbf{p}}\left(p_{\mathbf{p}}, Q_{\mathbf{s}} p_{\mathbf{p}} /\left(2 Q_{\mathbf{p}}\right)\right)$.

\section{CONCLUSIONS AND FUTURE WORK}

In this paper, a model that using Stackelberg game to maximize the profits of network operators including both $\mathrm{PO}$ and $\mathrm{SO}$ is proposed. It is considered first the changes of users' number which they choose operators based on their basic willingness to pay for each bandwidth. Then the specific process of the game are given. In addition, Analysis is conducted to verify the real-time of equilibrium pricing point and the correctness of the proposed method. At the Stackelberg equilibrium point, the following conclusions can be made: 1) The profits of both $\mathrm{PO}$ and $\mathrm{SO}$ are maximized. 2) Neither the user has incentive to change its operator, nor the operator has the incentive to change its price. 3) Our equilibrium pricing point is real-time with users' demands and network supplies.

The important factors that $p, b$ and the basic $\operatorname{cost} C_{\mathrm{p}}, C_{\mathrm{s}}$ will be considered to maximize the profits of operators at future work. The variables $p$ and $b$ reflect further the relationship between PO and SO as the leader and the follower.

\section{REFERENCES}

[1] Mark A, Mchenry, and Dan Mccloskey. Spectrum occupancy measurements: Chicago, illinois. technical report, Shared Spectrum Company, pages 16-18, November 2005.

[2] G. Faulhaber and D. Farber. Spectrum management: Property rights, markets and the commons. In Processing. Telecomm. Policy Research Conference, October 2003.
[3] Martin Cave, Chris Doyle, and William. Webb. Essentials of modern spectrum management. 2007.

[4] D. Varoutas, D. Katsianis, TH. Sphicopoulos, K. Stordahl, and I. Welling. On the economics of $3 \mathrm{~g}$ mobile virtual network operators (mvnos). Wireless Personal Communications, 36(2):129-142, January 2006.

[5] Lingjie Duan, Jianwei Huang, and Biying Shou. Investment and pricing with spectrum uncertainty: A cognitive operator's perspective. IEEE Transactions on mobile computing, 10(11):1590-1604, November 2011.

[6] S. K. Jayaweera and T. Li. Dynamic spectrum leasing in cognitive radio networks via primary-secondary user power control games. IEEE Transactions on Wireless Communications, 8(6):3300-3310, June 2009.

[7] E. Rasmusen. Games and information: An introduction to game theory. Wiley-Blackwell, November 2007.

[8] Shamik Sengupta, Mainak Chatterjee, and Samrat Ganguly. An economic framework for spectrum allocation and service pricing with competitive wireless service providers. In New Frontiers in Dynamic Spectrum Access Networks, 200\%. DySPAN 200\%. 2nd IEEE International Symposium on, pages 89-98, April 2007.

[9] Patrick. Maille Luis. Guijarro, Pla. Bruo. Tuffin and Jose. R. Vidal. Competition and bargaining in wireless networks with spectrum leasing. In Proceeding. IEEE Global Communications Conference (GLOBEALCOM 2011), pages 5-9, December 2011.

[10] D. Fudenberg and J. Tirole. Game Theory. MIT Press, Cambridge, MA, USA, 1991.

[11] Luis Guijarro, Vicent Pla, Jose R. Vidal, and Jorge Martinez-Bauset. Entry, competition, and regulation in cognitive radio scenarios: A simple game theory model. Mathematical Problems in Engineering, pages 1-13, December 2012.

[12] O. Simeone, I. Stanojev, S. Savazzi, Y. Bar-Ness, U. Spagnolini, and R. Pickholtz. Spectrum leasing to cooperating secondary ad hoc networks. IEEE Journal on Selected Areas in Communications, 26(1):203-213, January 2008.

[13] Cheng Zhang, BO Gu, Kyoko Yamori, Sugang Xu, and Yoshiaki Tanaka. Duopoly competition in time-dependent pricing for improving revenue of network service providers. IEICE Transactions on Communications, E96-B(12):2964-2975, December 2013.

[14] D. Niyato and E. Hossain. Competitive pricing for spectrum sharing in cognitive radio networks: Dynamic game, inefficiency of nash equilibrium, and collusion. IEEE Journal on Selected Areas in Communications, 26(1):192-202, 2008. 